\title{
Modulation of ionizing radiation-induced damage in human blood lymphocytes by in vivo treatment with resveratrol
}

\section{Eliana Evelina Ocolotobiche, Yuliana Catalina Banegas \& Alba Mabel Güerci}

To cite this article: Eliana Evelina Ocolotobiche, Yuliana Catalina Banegas \& Alba Mabel Güerci (2019): Modulation of ionizing radiation-induced damage in human blood lymphocytes by in vivo treatment with resveratrol, International Journal of Radiation Biology, DOI:

10.1080/09553002.2019.1625489

To link to this article: https://doi.org/10.1080/09553002.2019.1625489

Accepted author version posted online: 29

May 2019.

Submit your article to this journal $₫$

View Crossmark data \lceil 
Modulation of ionizing radiation-induced damage in human blood lymphocytes by in vivo treatment with resveratrol

Eliana Evelina Ocolotobiche ${ }^{\text {a,b,c }}$, Yuliana Catalina Banegas ${ }^{\text {a,c }}$, Alba Mabel Güerci ${ }^{\text {a,b,c* } *}$

a IGEVET - Instituto de Genética Veterinaria “Ing. Fernando N. Dulout” (UNLP-CONICET LA PLATA), Facultad de Ciencias Veterinarias, Universidad Nacional de Lâ Plata, Calle 60 y 118 s/n (CP 1900) La Plata, Buenos Aires, Argentina.

${ }^{\mathrm{b}}$ Facultad de Ciencias Exactas, Universidad Nacional de La Plata, Calle 47 y 115 s/n (CP 1900) La Plata, Buenos Aires, Argentina.

${ }^{c}$ Terapia Radiante S.A. Red CIO - La Plata. Calle 60 No 480 (CP 1900) La Plata, Buenos Aires, Argentina.

*corresponding author. E-mail: albaguerci@gmail.com 


\section{ABSTRACT}

Purpose: Radiotherapy is an effective tool for cancer control, but side effects on normal tissue limit its therapeutic effectiveness. Thus, the search for agents that may allow the use of high doses of radiation but exerting a differential protection to healthy tissue is of current concern. Resveratrol (3, 5, 4'-trihydroxy-trans-stilbene) (RSV) is a polyphenol with pleiotropic benefits for health due to its antioxidant and anti-inflammatory properties. Recent findings suggest that RSV could be promising in the fight against cancer since it inhibits the growth of tumor cells and optimizes radiotherapy. However, evidence in rodents and human beings is inconsistent. The aim of this study was to evaluate the radiomodulatory capacity of RSV on human lymphocytes.

Materials and methods: To study these properties of RSV, human peripheral blood lymphocytes from 20 healthy women undergoing in vivo RSV treatment with $50 \mathrm{mg} / \mathrm{day}$ doses were irradiated. The genotoxic damage was assessed by the comet assay, also called single cell gel electrophoresis (it makes it possible to measure the extent of the DNA migration from individual cells, detecting the genomic damage present in each cell).

Results: No differences were observed in basal clastogenic damage among samples without irradiation. There was only a slight radiation-induced clastogenic damage. The Damage Index (DI) value had a statistically significant increase in the exposed groups in comparison with the control groups $(\mathrm{p}<0.0001)$, but a statistically significant decrease of the DI value was observed in samples irradiated after treatment with RSV compared to pre-treatment samples $(\mathrm{p}<0.0001)$.

Conclusion: The RSV used as a dietary supplement had radioprotective properties, without exerting cytotoxic effect. The potential utility of RSV to optimize the radiotherapeutic ratio in cancer treatments using radiotherapy should be considered. 
Keywords: Resveratrol, Radiation, Radio-modulators, Radioprotection, in vivo

\section{Introduction}

Radiotherapy is a common therapeutic modality in modern medicine based on the use of ionizing radiation for cancer treatment (United Nations Scientific Committee on the Effects of Atomic Radiation 2008; Barnett et al. 2015). Although radiation is effective for disease control, side effects such as damage in normal tissues surrounding the tumor limit its therapeutic benefits (West and Barnett 2011; Raabe et al. 2012). Recent advances have focused on achieving an optimal balance between tumoricidal efficacy and acceptable toxic effects. Usually, radiotherapy is performed using many relatively small fractions of radiation, but currently, there is a push to increase overall total dose and to increase in fraction size mostly taking advantage of more accurate delivery thus avoiding or giving less dose to the surrounding normal tissue. Therefore, there is interest in protecting the normal tissue, so research is centered in finding agents that exerts a differential protection to healthy tissue. Since cellular damage induced by electromagnetic radiation is mainly attributed to the harmful effect of free radicals (Zhang et al. 2013; Li et al. 2014; Koohian et al. 2017), substances that could eliminate them are particularly attractive as radio-modulators. A large number of natural and synthetic compounds such as antioxidants, cytoprotective agents and vitamins have been extensively studied in in vitro and in vivo models (Brisdelli et al. 2009). Unfortunately, some radioprotectors are toxic at the doses required for this end, and many synthetic agents have not been used in clinical applications yet. In addition, the search for new agents for cancer prevention and treatment has been strengthened in recent years due to the progressive increase of the disease as a result of both life style changes and increased longevity (GLOBOCAN 2018). Natural products have always been an important source of these new agents; thus, numerous studies have focused on biologically active substances of plant origin having therapeutic properties (Kundu and Surh 2008; Shukla and Singh 2011). 
Resveratrol (3,5,4'-trihydroxy-trans-stilbene) (RSV) is a polyphenol commonly obtained from grapes and their derivatives and also synthesized by several plant species in response to biotic and abiotic stress. The compound has shown pleiotropic benefits for health, particularly because of its antioxidant, anti-inflammatory, anti-aging, cardioprotective and neuroprotective activities (Brisdelli et al. 2009; Shukla and Singh 2011; Carter et al. 2014; Poulsen et al. 2015). Considering that some of these properties are supposed to counteract both the carcinogenic process and the side effects of the radiant protocols, much of the current research on RSV is concerned with its possible application in oncology as a therapeutic and/or chemopreventive agent (Singh CK et al. 2015). In this sense, recent findings from our laboratory and other research groups suggest that RSV could be promising in the fight against cancer as well as in the improvement of radiation therapy (Kundu and Surh 2008; Brisdelli et al. 2009; Carter et al. 2014). As a polyphenolic compound, RSV removes hydroxyl and superoxide radicals and promotes the activity of antioxidant enzymes such as superoxide dismutase and catalase. Their reduction prevents DNA damage, which could be useful in oncological radiotherapy to avoid normal tissue damage (Brisdelli et al. 2009).

On the other hand, recent work has remarkably advanced our understanding of the molecular mechanisms underlying RSV anticancer properties. For instance, it has been shown to inhibit or retard the growth of tumor cells in vitro and tumors implanted in vivo (Brisdelli et al. 2009; Banegas et al. 2018). However, although the data obtained from cell cultures are promising, evidence in rodents and human beings is scant and inconsistent. Contradictory results may be due to the route of administration and the dose or animal species used, among other factors. Thus, the transfer of RSV to the clinical area is still far from real considering the challenges to be addressed, namely, from metabolic issues to the total understanding of RSV systemic action (Shukla and Singh 2011; Singh CK et al. 2015). 
According to the above mentioned and previous results obtained by our research group (Banegas et al. 2018), the aim of this study was to evaluate the radiomodulatory capacity of RSV in 4 Gy-irradiated human peripheral blood lymphocytes from healthy women undergoing in vivo RSV treatment.

\section{Materials and methods}

\subsection{Chemicals}

Low-melting-point agarose (LMA), normal-melting point agarose (NMA), $\mathrm{NaCl}, \mathrm{Na}-$ EDTA, Tris, NaOH, Triton X-100, ethanol, EDTA and dimethyl sulfoxide (DMSO) were obtained from Invitrogen (California, USA), Biopack (Buenos Aires, Argentina) and Carlo Erba Reagents (Barcelona, Spain). Resveratrol capsules were purchased from N.S. Products S.R.L. (Buenos Aires, Argentina), RPMI 1640 Medium from Gibco (California, USA) and Sybr green from Sigma-Aldrich (Missouri, USA)

\subsection{Study design}

The experimental group was composed of healthy, non-smoking female volunteers with similar nutritional conditions. Participants received one capsule of $50 \mathrm{mg}$ RSV daily under fasting conditions for 15 consecutive days. A sample of about $5 \mathrm{~mL}$ peripheral blood was drawn in labelled tubes containing lithium heparin as anticoagulant (Monovettes®; Sarstedt, Nümbrecht, Germany) at the beginning and end of this treatment. Immediately afterwards, two aliquots of blood ( $1 \mathrm{~mL}$ each) were separated and centrifuged for $5 \mathrm{~min}$ at $1000 \mathrm{rpm}$ at $20{ }^{\circ} \mathrm{C}$. Plasma was removed and replaced for an equal volume of RPMI, and the pellets were resuspended. One aliquot was irradiated with $4 \mathrm{~Gy}$ and the other was used as experimental control. Genotoxic damage was assessed with the alkaline comet assay. 
The study was performed in accordance with the core principles of the Good Clinical Practice Guidelines (Vijayananthan and Nawawi 2008) and following the principles of the Declaration of Helsinki. All donors were properly informed of the purposes of this study and gave their written informed consent.

A total of 20 healthy female subjects (age range, 20-30 years) were recruited. General characteristics of the study sample are presented in Table 1. Eligibility criteria met the following requirements: normal physical examination, vital signs and laboratory screening results within normal ranges, willingness to abstain from specific foodstuff and beverages, ability to understand the full nature and purpose of the study, non-smoking, non-pregnant and non-lactating women (Sergides et al. 2016). Exclusion criteria included history of hypersensitivity to the test substance and the inactive ingredients, hospitalization for any reason or blood donation $(\geq 450 \mathrm{ml}$ ) within 12 weeks prior to the initiation of the study, intake of any drugs within 4 weeks prior to or during the study, history or presence of any relevant medical condition, history of drug or alcohol abuse, and subjects that were vegetarian or followed a particular diet.

From three days prior to the study and up to $24 \mathrm{~h}$ after the last RSV dose, volunteers maintained a strict diet, avoiding the consumption of foods or beverages with a high RSV content such as red/black fruit, kiwi fruit, red/black grapes, peanuts, nuts and red wine. The diet was controlled during the entire treatment period. Each RSV tablet was administered orally with $200 \mathrm{ml}$ water early in the morning on an empty stomach.

\subsection{Irradiation procedure}

Cell irradiation was performed with a VarianClinac $®$ 6MV linear accelerator (Varian Medical Systems, Palo Alto, California, USA). The dose rate was calculated using isocentric formalism and set at $300 \mathrm{cGy} / \mathrm{min}$. The $1.5 \mathrm{ml}$ tubes containing $1 \mathrm{ml}$ of sample were placed 
in an immobilized rack inside an acrylic phantom with water, whose density was equivalent to soft tissue and whose depth was greater than the buildup zone. This system was placed on a "tennis racket" support panel of the accelerator and irradiated from the bottom with 4 Gy isocentric photon beams using a 10-15 X field size. The deviation of the absorbed dose was less than 5\%, which was compatible with the therapeutic objective. The samples were irradiated at room temperature, but immediately after that they were put on ice. Despite for conventional radiotherapy treatments the exposure is usually given on 2 Gray fractions once per day five days per week, previous results obtained in our laboratory showed that 4 Gy was optimal in relation to the sensitivity of the technique used (Olive 2009; Banegas et al. 2018).

\subsection{Comet assay}

Cytomolecular genotoxic damage in peripheral blood was assessed immediately after each radiation treatment with the alkaline version of the comet assay according to Singh NP et al. (1988) with slight modifications. Microscopic slides were coated with NMA and kept at room temperature for agarose gel solidification. Twenty microliters of treated cells were mixed with $160 \mu \mathrm{L}$ LMA in phosphate-buffered saline; $90 \mu 1$ of the mixture were layered on each slide, spread out with a cover slip and kept at $4{ }^{\circ} \mathrm{C}$ for 10 min. After solidification, coverslips were removed and slides were immersed in cold lysing solution $(2.5 \mathrm{M} \mathrm{NaCl}, 100$ $\mathrm{mM} \mathrm{Na} 2 \mathrm{EDTA}, 10 \mathrm{mM}$ Tris, $\mathrm{pH}$ 10); then, $1 \%$ Triton $\mathrm{X}-100$ and 10\% DMSO were added just before use. After lysis, the slides were placed in a horizontal gel electrophoresis tank filled with alkaline buffer (300 mM NaOH and $1 \mathrm{mM}$ EDTA, $\mathrm{pH} 13)$ for $20 \mathrm{~min}$ at $4{ }^{\circ} \mathrm{C}$ to allow DNA unwinding and the expression of alkali-labile damage. Electrophoresis was performed for $25 \mathrm{~min}$ at $250 \mathrm{~mA}$ and $25 \mathrm{~V}$. After electrophoresis, the slides were washed three times for 5 min with neutralizing buffer ( $0.4 \mathrm{M}$ Tris, $\mathrm{pH} 7.5)$ and fixed with ethanol absolute. 


\subsection{Microscopic analysis and scoring}

Slides were scored under blind code. They were stained with $20 \mu 1$ Sybr green $(1 / 2000$ dilution) just before the microscopic analysis. A total of 100 randomly captured cells per slide were visually analyzed under a fluorescence microscope with excitation filters of 515$560 \eta \mathrm{m}$ (Olympus BX40®, Tokyo, Japan) at 400 magnification. Each experimental point was evaluated in duplicate. Thus, total cell observations included 800 cells per volunteer (200 cells per condition). The degree of damage was determined visually according to Collins et al. (1995) (Kumaravel et al. 2009). Each cell was classified into 5 classes, from class 0 (no DNA migration) to class 4 (maximum DNA migration). Genetic damage was measured with the damage index $(D I)$, calculated with the formula DI $=[(1 \times n 1)+(2 \times n 2)+(3 \times n 3)+(4 \times$ $\mathrm{n} 4)] /(\mathrm{n} 0+\mathrm{n} 1+\mathrm{n} 2+\mathrm{n} 3+\mathrm{n} 4) \times 100$, where $n$ indicates the number of cells in each class. Damage class (DC) was calculated as the sum of cells with 0 (null), 1 and 2 (slight), and 3 and 4 (severe) damage.

\subsection{Statistical analysis}

Data were analyzed using Student's t-test and Chi-square test. Results are expressed as means \pm SEM.

\section{Results}

All volunteers completed the study. The demographic characteristics of participants are presented in Table 1 (mean age, $25.39 \pm 2.44$ years [range, 22 - 30 years]; mean weight, $60.43 \pm 6.018 \mathrm{~kg}$ [range, 49.2-76.0 kg); mean height, $1.62 \pm 0.06 \mathrm{~m}$ [range, 1.48-1.76 m]; mean Body Mass Index kg/m², $22.98 \pm 2.04$ [range, 19.33-29.69]). [Table 1 near here] 
Mean frequency of comet classes for each experimental point is shown in Table 2. Comet classes 3 and 4 were observed more frequently in the irradiated than in the control groups, while comet class 0 was observed more frequently in controls. [Table 2 near here]

Figure 1A shows DC of blood samples taken before and after daily RSV administration for 15 days. No significant differences were observed in basal clastogenic damage among control, pre- and post-treatment samples without irradiation, suggesting that both RSV and its metabolites did not exert a genotoxic effect at the dose administered. Despite the absence of unwanted side effects in volunteers, our results failed to verify the antioxidant capacity of RSV. Radiation-induced clastogenic damage comparing irradiated cells (4 Gy) before and after treatment, was slight and mostly corresponded to comet grades 2 and 3 (Figure 1A). This tendency was repeated in all volunteers. Figure 1B shows the DI of blood samples. While the lowest DI value was observed in the control groups (not irradiated), both before (mean DI 24,72 $\pm 6,42$ ) and after (mean DI 19,07 $\pm 4,05$ ) treatment with RSV, this parameter had a significant increase in the exposed groups $(\mathrm{p}<0.0001)$. An important DI decrease $(\mathrm{p}<0.0001)$ was observed in samples irradiated after treatment with RSV (mean DI 120,52 \pm 9,22 ) compared to pre-treatment samples (mean DI $171,91 \pm 6,44$ ), it was noted that the DI increased with the irradiation and decreased with the RSV treatment. [Figure 1 near here]

\section{Discussion}

Currently, radiotherapy is the cornerstone of cancer treatment considering that it is a targeted and non-invasive therapy, with good organ preservation. However, its side effects on normal tissue limit its use, making it necessary to find agents to mitigate them (Citrin et al. 2010). Although radiotoxicity would be related to immediate and widespread oxidative damage to the genetic material of irradiated cells, new paradigms propose broader mechanisms of action which are integrated at systemic level (Güerci and Córdoba 2015). In 
general, cell damage has been attributed to the increase in reactive oxygen species that induce functional alterations in lipids, proteins and nucleic acids (Zhang et al. 2013; Li et al. 2014; Dobrzynska et al. 2016). Thus, molecules with free radical scavenging properties are particularly promising as radio protectors, and their clinical use has been investigated during the last years. Since these compounds frequently have side effects and toxicity (Sebastia et al. 2013; Li et al. 2014), the focus is now being placed on natural compounds with lower toxicity than the synthetic substances and with most favorable administration routes (Sebastia et al. 2013). Among them, RSV has been highly promoted both as antioxidant and anticancer (Shukla and Singh 2011; Zhang et al. 2013). Such dual mechanism of action constitutes the fundamental basis of a radio-protective substance, i.e., protective for normal cells and toxic for tumor cells (Fabre et al. 2011; Sebastia et al. 2014).

Taking into account the tissue analyzed in this study (human peripheral blood), an important aspect to consider is that the side effects of radiotherapy vary depending on the area treated, the general condition of the patient and the type and dose of radiation used. Treatment over large areas of the body, such as bones that contain a main part of the bone marrow, for example pelvis, legs, thorax, or abdomen, may lead to drop of the white and red blood cell count (American Cancer Society 2019; American Society of Clinical Oncology 2017). In relation, it is suggested that special treatments, such as Total Body Irradiation, induce significant leukopenia. It has also been observed a reduction in the number of circulating leukocytes and lymphocytes in cervical and endometrium radiotherapy (van Meir et al. 2017). In this way and in agreement with Yi et al. (2019), there is a need to consider agents, such as resveratrol, that provide protection against hematopoietic injury induced by irradiation. On the other hand, currently relevant protocols like hypofractionated radiotherapy, induce damage on infiltrating lymphocytes (Arnold et al. 2018), and could also be optimized using substances with radioprotective properties. 
Most studies related to the possible application of RSV in oncological therapy have been carried out in in vitro models. Results of in vivo treatments are scarce, and evidence for their clinical use in human beings is still inconsistent (Carter et al. 2014) even more when administered along with other therapies. Thus, we evaluated the effectiveness of RSV against radiation-induced genetic damage after in vivo treatments and studied its genotoxicity in human lymphocytes to optimize the most widely used oncological treatment these days.

Previous studies from our group (Banegas et al. 2018) and other authors (Zhang et al. 2013; Sebastia et al. 2014; Meng et al. 2016) indicate that the chemo-protective effect of RSV depends on the dose administered and the synergistic interaction with other drugs or cytotoxic factors. Accordingly, the dose recommended as a dietary supplement (50 mg/day) and that showed results in mice (Koohian et al. 2017) was chosen, considering that doses of 2 $\mathrm{mg} / \mathrm{kg} / \mathrm{day}$ in human beings would exercise radioprotection and that there was no toxicity from $25 \mathrm{mg}$ to $5 \mathrm{~g} / \mathrm{day}$. In this sense, our results could experimentally guarantee that treatment of young and healthy women with such dose had good systemic tolerance and no stomach discomfort, dizziness, fatigue, headache or cutaneous reactions.

Genotoxic damage and the radiomodulatory potential of RSV were analyzed with the comet assay, taking into account its high sensitivity to detect and quantify DNA damage induced by several agents and by ionizing radiation. This test allowed the analysis of DNA strand breaks induced by x-irradiation in the presence or absence of RSV. The entero-hepatic recirculation of the compound enabled its slow elimination while allowing prolonged effects (Delmas et al. 2006), being it therefore plausible to detect it in the post-treatment times evaluated.

The fact that no significant differences were observed in cytomolecular DI before and after in vivo treatment with RSV suggests that RSV and its secondary metabolites did not exert clastogenic effects on human peripheral lymphocyte DNA at the dose administered. 
Thus, the interference of RSV with topoisomerases and increased DNA damage attributed to RSV (Sebastia et al. 2013) could not be demonstrated. On the other hand, RSV radioprotective effect against ionizing radiation-induced genotoxicity could be seen. In our study, pretreatment with RSV decreased DNA strand breaks in irradiated human lymphocytes, which agrees with Carsten et al. (2008), who endorsed that oral RSV administration to mice acted as a generalized radioprotector, decreasing the frequency of chromosomal aberrations induced by radiation in the same cells. Our observation that treatment with RSV decreased DI by $29.41 \%$ in irradiated lymphocytes clearly indicated that RSV prevented radio-induced DNA damage. This is also in agreement with Koohian et al. (2017), who found that RSV offered the best protection with low toxicity against 2 Gy irradiation in mice evaluating the effects by the comet assay.

Taking the genotoxicity and radiomodulation analyses of this work together, in vitro evidence also showed that although trans-resveratrol decreased the damage in blood lymphocytes exposed to 2 Gy X-rays, few chromosomal aberrations were found when these cells were treated with RSV without being exposed to radiation (Sebastia et al. 2013; Sebastia et al. 2014; Dobrzynska et al. 2016). On the topic of the mechanisms of action, RSV is a hydrophobic compound able to cross cell membranes and eliminate radio-induced free radicals that are close to the DNA double helix and alter its structure (Koohian et al. 2017). Thus, RSV is able not only to scavenge these free radicals but also to maintain and restore the levels of intra-cellular antioxidants (Carsten et al. 2008; Sebastia et al. 2013; Zhang et al. 2013). At molecular level, RSV decreased the effects of radiation at least partly based on Sirt1 expression and activity (Zhang et al. 2013; Li et al. 2014). This protein plays an important role in oxidative stress, cell proliferation and genomic stability, among other biological processes. An increase in Sirtl mRNA and in protein activity has been demonstrated after treatment with RSV. 
Ultimately, it should be noted that RSV systemic impact would also be effective in relation to the holistic effect of irradiations, since it exhibits anti-inflammatory activity through the modulation of enzymes and mediators of inflammation (Kundu and Surh 2008; Udenigwe et al. 2008), and also enhances the immune response against cancer (Soldati et al. 2018), both central elements of the response to radiant treatment.

\section{Conclusions}

Our results suggest that RSV could be a successful radiomodulator to protect human lymphocytes from the harmful effects of irradiation. Daily doses of $50 \mathrm{mg}$ did not show genotoxic risk, nor did they present adverse effects that may generate controversies among doctors or patient concern. Administration of RSV could help to protect against genomic instability and eventual radio-induced carcinogenesis, as well as against the side effects of radiant treatments. In this way, radiotherapy, as well as other oncological treatments, diseases, certain medications and poor nutrition, can affect white blood cells, weakening the immune system. This deficit of white blood cells that sensitizes the individual to an infection could be counteracted by the supply of appropriate substances such as RSV. The good pharmacological availability and affordable cost position RSV as a good candidate to optimize the radiotherapeutic quotient promoting the elimination of cancer cells subject to radiotherapy and decreasing chronic oxidative stress and inflammation responsible for the side effects of this treatment.

\section{Conflict of interest}

The authors declare no conflict of interest. 


\section{Acknowledgements}

The authors thank Marcelo Martínez for assistance in the design of the irradiation treatment and dose calculations, and the authorities of the Institute of Radiant Therapy network (CIO), La Plata. Thanks are also due to A. Di Maggio for manuscript correction and editing.

\section{References}

American Cancer Society. 2019. Radiation Therapy for Endometrial Cancer. Retrieved from https://www.cancer.org/cancer/endometrial-cancer/treating/radiation.html

American Society of Clinical Oncology. 2017. Uterine Cancer: Treatment Options. Retrieved from https://www.cancer.net/cancer-types/uterine-cancer/treatment-options

Arnold KM, Flynn NJ, Raben A, Romak L, Yu Y, Dicker AP, Mourtada F, Sims-Mourtada J. 2018. The Impact of Radiation on the Tumor Microenvironment: Effect of Dose and Fractionation Schedules. Cancer Growth Metastasis 11:1-17

Banegas YC, Ocolotobiche EE, Padula G, Córdoba EE, Fernández E, Güerci AM. 2018. Evaluation of resveratrol radiomodifying potential for radiotherapy treatment. Mutat Res Gen Tox En. 836:79-83

Barnett GC, Kerns SL, Noble DJ, Dunning AM, West CM, Burnet NG. 2015. Incorporating Genetic Biomarkers into Predictive Models of Normal Tissue Toxicity. Clin Oncol (R Coll Radiol). 27(10):579587.

Brisdelli F, D'Andrea G, Bozzi A. 2009. Resveratrol: a natural polyphenol with multiple chemopreventive properties. Curr Drug Metab. 10(6):530-546.

Carsten RE, Bachand AM, Bailey SM, Ullrich RL. 2008. Resveratrol reduces radiation-induced chromosome aberration frequencies in mouse bone marrow cells. Radiat Res. 169(6):633-638.

Carter LG, D'Orazio JA, Pearson KJ. 2014. Resveratrol and cancer: focus on in vivo evidence. Endocr Relat Cancer. 21(3):R209-225.

Citrin D, Cotrim AP, Hyodo F, Baum BJ, Krishna MC, Mitchell JB. 2010. Radioprotectors and mitigators of radiation-induced normal tissue injury. Oncologist. 15(4):360-371.

Collins AR, Ma AG, Duthie SJ. 1995. The kinetics of repair of oxidative DNA damage (strand breaks and oxidized pyrimidine) in human cells. Mutat Res. 336:69-77.

Delmas D, Lancon A, Colin D, Jannin B, Latruffe N. 2006. Resveratrol as a chemopreventive agent: a promising molecule for fighting cancer. Curr Drug Targets. 7(4):423-442.

Dobrzynska MM, Gajowik A, Radzikowska J. 2016. The effect of in vivo resveratrol supplementation in irradiated mice on the induction of micronuclei in peripheral blood and bone marrow reticulocytes. Mutagenesis. 31(4):393-399.

Fabre KM, Saito K, DeGraff W, Sowers AL, Thetford A, Cook JA, Krishna MC, Mitchell JB. 2011. The effects of resveratrol and selected metabolites on the radiation and antioxidant response. Cancer Biol Ther. 12(10):915-923.

GLOBOCAN, IARC Global Cancer Observatory. 2018. Latest global cancer data: Cancer burden rises to 18.1 million new cases and 9.6 million cancer deaths in 2018. PRESS RELEASE Nº 263. 
Güerci AM, Córdoba EE. 2015. Nuevo Enfoque de los efectos biológicos de las radiaciones. Revista Argentina de Radiobiología. 79(4):224-225.

Koohian F, Shanei A, Shahbazi-Gahrouei D, Hejazi SH, Moradi MT. 2017. The Radioprotective Effect of Resveratrol Against Genotoxicity Induced by gamma-Irradiation in Mice Blood Lymphocytes. Dose Response. 15(2).

Kumaravel TS, Vilhar B, Faux SP, Jha AN. 2009. Comet Assay measurements: a perspective. Cell Biol Toxicol. 25:53-64.

Kundu JK, Surh YJ. 2008. Cancer chemopreventive and therapeutic potential of resveratrol: mechanistic perspectives. Cancer Lett. 269(2):243-261.

Li J, Feng L, Xing Y, Wang Y, Du L, Xu C, Cao J, Wang Q, Fan S, Liu Q et al. 2014. Radioprotective and antioxidant effect of resveratrol in hippocampus by activating Sirt1. Int J Mol Sci. 15(4):5928-5939.

Meng J, Guo F, Xu H, Liang W, Wang C, Yang XD. 2016. Combination Therapy using Co-encapsulated Resveratrol and Paclitaxel in Liposomes for Drug Resistance Reversal in Breast Cancer Cells in vivo. Sci Rep. 6:22390.

Olive PL. 2009. Impact of the comet assay in radiobiology. Mutat Res. 681(1):13-23.

Poulsen MM, Fjeldborg K, Ornstrup MJ, Kjaer TN, Nohr MK, Pedersen SB. 2015. Resveratrol and inflammation: Challenges in translating pre-clinical findings to improved patient outcomes. Biochim Biophys Acta. 1852(6):1124-1136.

Raabe A, Derda K, Reuther S, Szymczak S, Borgmann K, Hoeller U, Ziegler A, Petersen C, Dikomey E. 2012. Association of single nucleotide polymorphisms in the genes ATM, GSTP1, SOD2, TGFB1, XPD and XRCC1 with risk of severe erythema after breast conserving radiotherapy. Radiat Oncol. 7:65.

Sebastia N, Almonacid M, Villaescusa JI, Cervera J, Such E, Silla MA, Soriano JM, Montoro A. 2013. Radioprotective activity and cytogenetic effect of resveratrol in human lymphocytes: an in vitro evaluation. Food Chem Toxicol. 51:391-395.

Sebastia N, Montoro A, Hervas D, Pantelias G, Hatzi VI, Soriano JM, Villaescusa JI, Terzoudi GI. 2014. Curcumin and trans-resveratrol exert cell cycle-dependent radioprotective or radiosensitizing effects as elucidated by the PCC and G2-assay. Mutat Res. 766-767:49-55.

Sergides C, Chirila M, Silvestro L, Pitta D, Pittas A. 2016. Bioavailability and safety study of resveratrol $500 \mathrm{mg}$ tablets in healthy male and female volunteers. Exp Ther Med. 11(1):164-170.

Shukla Y, Singh R. 2011. Resveratrol and cellular mechanisms of cancer prevention. Ann N Y Acad Sci. 1215:1-8.

Singh CK, Liu X, Ahmad N. 2015. Resveratrol, in its natural combination in whole grape, for health promotion and disease management. Ann N Y Acad Sci. 1348(1):150-160.

Singh NP, McCoy MT, Tice RR, Schneider EL. 1988. A simple technique for quantitation of low levels of DNA damage in individual cells. Exp Cell Res. 175(1):184-191.

Soldati L, Di Renzo L, Jirillo E, Ascierto PA, Marincola FM, De Lorenzo A. 2018. The influence of diet on anti-cancer immune responsiveness. J Transl Med. 16(1):75.

Udenigwe CC, Ramprasath VR, Aluko RE, Jones PJ. 2008. Potential of resveratrol in anticancer and anti-inflammatory therapy. Nutr Rev. 66(8):445-454.

United Nations Scientific Committee on the Effects of Atomic Radiation. 2008. Sources and effects of ionizing radiation. Report to the General Assembly with scientific annexes. $1 \mathrm{~A}$.

van Meir H, Nout RA, Welters MJP, Loof NM, de Kam ML, van Ham JJ, Samuels S, Kenter GG, Cohen AF, Melief CJM, Burggraaf J, van Poelgeest MIE, and van der Burg SH. 2017. Impact of (chemo)radiotherapy on immune cell composition and function in cervical cancer patients. Oncoimmunology. Vol. 6, No. 2, e1267095.

Vijayananthan A, Nawawi O. 2008. The importance of Good Clinical Practice guidelines and its role in clinical trials. Biomed Imaging Interv J. 4(1):e5.

West CM, Barnett GC. 2011. Genetics and genomics of radiotherapy toxicity: towards prediction. Genome Med. 3(8):52. 
Yi L, Tian M, Piao C, Gao G, Wu L, Pan Y, Li J. 2019.The protective effects of 1,2-propanediol against radiation-inducedhematopoietic injury in mice. Biomedicine \& Pharmacotherapy journal. Vol. 114,e 108806

Zhang $\mathrm{H}$, Zhai Z, Wang Y, Zhang J, Wu H, Wang Y, Li C, Li D, Lu L, Wang X et al. 2013. Resveratrol ameliorates ionizing irradiation-induced long-term hematopoietic stem cell injury in mice. Free Radic Biol Med. 54:40-50.

(A) Damage Class

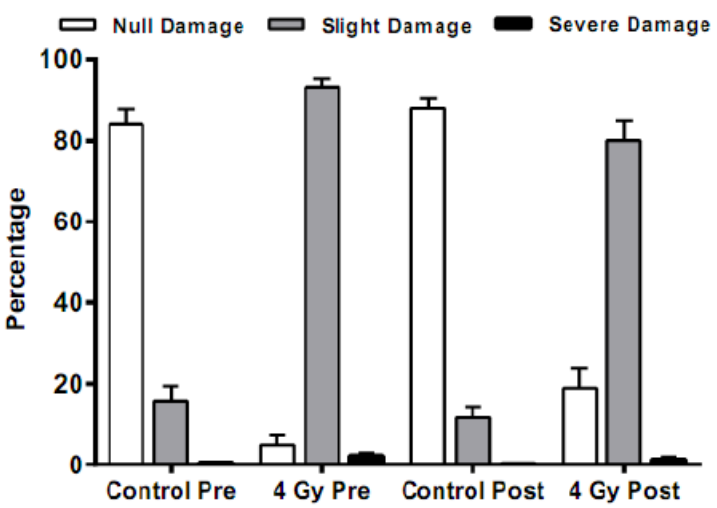

(B)

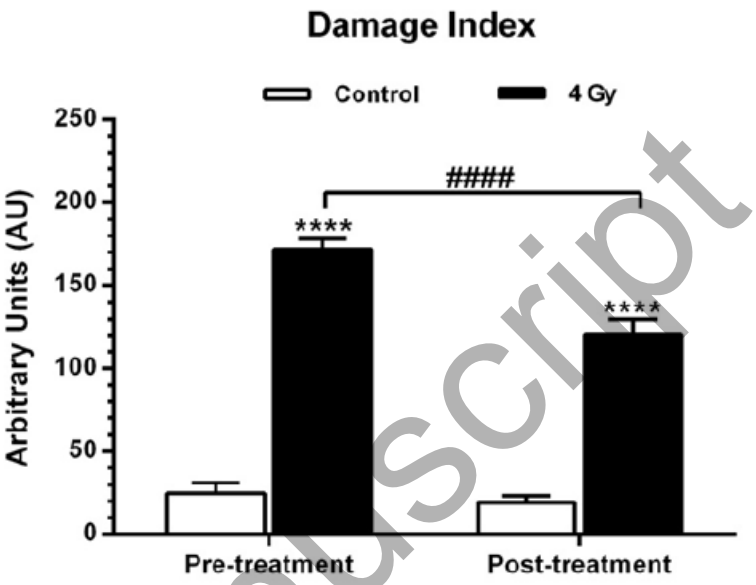

Figure 1. Radiation-induced genotoxic effect evaluated in human peripheral blood lymphocytes pre and post-treatment with RSV. Panel A: Clastogenic damage class for each of the analyzed points, expressed as mean \pm SEM of 20 samples in duplicate. Panel B: Damage index for each treatment performed, expressed as mean \pm SEM of 20 samples in duplicate. ${ }^{* * * *} p<0.0001$ control vs 4Gy, pre and post treatment. \#\#\#\# $\mathrm{p}<0.0001$ pre vs. post treatment irradiated with 4 Gy. One-way ANOVA and post-hoc Tukey's test. 
Table 1. Demographic characteristics of the study sample.

\begin{tabular}{|c|c|c|c|c|}
\hline Volunteer & Age & Weight (kg) & $\begin{array}{c}\text { Height } \\
\text { (m) }\end{array}$ & Body Mass Index $\left(\mathrm{kg} / \mathrm{m}^{2}\right)$ \\
\hline 1 & 23 & 64.600 & 1.60 & 25.234 \\
\hline 2 & 22 & 53.000 & 1.54 & 22.348 \\
\hline 3 & 24 & 70.000 & 1.64 & 26.026 \\
\hline 4 & 25 & 70.000 & 1.62 & 26.673 \\
\hline 5 & 24 & 61.500 & 1.61 & 23.726 \\
\hline 6 & 24 & 52.500 & 1.62 & 20.005 \\
\hline 7 & 22 & 60.000 & 1.55 & 24.974 \\
\hline 8 & 25 & 61.000 & 1.63 & \\
\hline 9 & 25 & 61.000 & 1.65 & \\
\hline 10 & 29 & 60.000 & 1.65 & 9 \\
\hline 11 & 24 & 63.000 & 1.60 & 24.609 \\
\hline 12 & 24 & 64.000 & 1.63 & 24.088 \\
\hline 13 & 25 & 60.000 & 1.60 & 23.438 \\
\hline 14 & 26 & 64.000 & 10 & 23.795 \\
\hline 15 & 30 & 52.000 & 1.64 & 19.334 \\
\hline 16 & 29 & 53.000 & 1.64 & 19.706 \\
\hline 17 & 28 & 63.000 & 1.65 & 23.140 \\
\hline 18 & 24 & 71.000 & 1.70 & 24.567 \\
\hline 19 & 27 & 62.000 & 1.76 & 20.015 \\
\hline 20 & 30 & 49.200 & 1.48 & 22.462 \\
\hline Range & $22-3$ & $49.2-76.0$ & $1.48-1.76$ & $19.33-29.69$ \\
\hline Mean & 25.39 & 60.435 & 1.62 & 22.98 \\
\hline SD & 4 & 6.018 & 0.06 & 2.04 \\
\hline
\end{tabular}


Table 2. Mean frequency of comet classes from 4000 cells $( \pm$ SEM) for each experimental point.

\begin{tabular}{lcccccccccc}
\hline & \multicolumn{2}{c}{ Class 0 } & \multicolumn{2}{c}{ Class 1 } & \multicolumn{2}{c}{ Class 2 } & \multicolumn{2}{c}{ Class 3 } & \multicolumn{2}{c}{ Class 4 } \\
& Mean & SEM & Mean & SEM & Mean & SEM & Mean & SEM & Mean & SEM \\
\hline Pre-treatment Control & 79.34 & 4.92 & 7.63 & 2.27 & 12.31 & 4.22 & 0.00 & 0.00 & 0.71 & 0.26 \\
Pre-treatment 4Gy & 4.80 & 2.36 & 22.06 & 3.09 & 70.97 & 4.23 & 0.37 & 0.19 & 1.80 & 0.56 \\
Post-treatment Control & 88.05 & 2.46 & 5.10 & 0.92 & 6.67 & 1.75 & 0.00 & 0.00 & 0.18 & 0.10 \\
Post-treatment 4Gy & 19.00 & 4.83 & 43.29 & 4.25 & 36.60 & 5.03 & 0.02 & 0.03 & 1.09 & 0.58 \\
\hline
\end{tabular}

